\title{
Postpartum anticoagulation in women with mechanical heart valves
}

This article was published in the following Dove Press journal:

International Journal of Women's Health

\author{
Roxanna A Irani ${ }^{1, *}$ \\ Ann Santa-Ines ${ }^{2, *}$ \\ Robert W Elder ${ }^{2}$ \\ Heather S Lipkind' \\ Michael J Paidas' \\ Katherine H Campbell' \\ 'Department of Obstetrics, \\ Gynecology and Reproductive \\ Sciences, Section of Maternal-Fetal \\ Medicine, Yale University School \\ of Medicine, New Haven, CT, USA; \\ ${ }^{2}$ Department of Pediatrics, Section of \\ Pediatric Cardiology, Adult Congenital \\ Heart Program, Yale University School \\ of Medicine, New Haven, CT, USA
}

*These authors contributed equally to this work
Correspondence: Roxanna A Irani Department of Obstetrics, Gynecology and Reproductive Sciences, Section of Maternal-Fetal Medicine, University of California San Francisco, 550 16th Street, 7th Floor, San Francisco, CA 94143, USA Tel +l 4I54763156

Email roxanna.irani@ucsf.edu
Background: Women with mechanical heart valves (MHV) requiring anticoagulation (AC) are at high risk for hemorrhagic complications. Despite guidelines to manage antenatal and peripartum AC, there are few evidence-based recommendations to guide the initiation of postpartum AC. We reviewed our institutional experience of pregnant women with MHV to lay the groundwork for recommendations of immediate postpartum AC therapy.

Study design: This descriptive retrospective cohort used ICD-9 and -10 codes to identify pregnant women with MHV on AC at the Yale-New Haven Hospital from 2007 to 2018. All identified patients were confirmed by chart review. Delivery hospitalization and the immediate postpartum AC management were reviewed. Maternal complications recorded were postpartum hemorrhage, transfusion, wound hematoma, intra-abdominal bleeding, stroke, valve thrombosis, and death. Further, immediate neonatal outcomes were detailed.

Results: Forty-two pregnant women with nonnative heart valves were identified during the study period. From those pregnant women, nine had an MHV and were anticoagulated throughout gestation. Of 19 total pregnancies, 14 met the inclusion criteria. The median gestational age of the delivered pregnancies was early term (37w2d). Nine deliveries were via cesarean (64\%). The median time to restart AC after birth was 6 hours. After six deliveries (43\%), AC was initiated $\leq 6$ hours postpartum. Hemorrhagic complications occurred in six cases $(43 \%)$, including wound and intra-abdominal hematomas. Four cases $(29 \%)$ required blood transfusion. No maternal strokes, thrombotic events, or deaths were recorded. Five (38.5\%) neonates required admission to the neonatal intensive care unit.

Conclusion: MHV in pregnancy was rare but was associated with significant maternal morbidity, particularly postpartum hemorrhagic complications. We noted significant variability in the timing of restarting postpartum AC and in the selected agents. Pooled institutional data and an interdisciplinary approach are recommended to minimize competing risks and sequelae of valve thrombosis and obstetrical hemorrhage and, thereby, to optimize maternal outcomes and develop evidence-based guidelines for postpartum AC management.

Keywords: pregnancy, anticoagulation, postpartum hemorrhage, mechanical heart valve

\section{Plain language summary}

Women with mechanical heart valves are at increased risk for various complications during pregnancy and in the postpartum period. Consistent use of anticoagulation is required to reduce the risk of a blood clot forming on the mechanical valve - a life-threatening emergency. The mother's anticoagulation is stopped during labor and delivery (or cesarean delivery) to reduce the risk of bleeding during childbirth. However, there is limited guidance on the best time to restart the anticoagulation after the birth of the baby. This study has revealed that there is a significant risk of bleeding complications in the postpartum period as well as wide variation on when the anticoagulation is restarted after delivery. Information obtained from this study will help provide guidance in developing a safer approach to start anticoagulants after childbirth. 
Initiation of anticoagulation after delivery should optimally minimize the risk of bleeding and the risk of blood clot formation.

\section{Introduction}

Over the past several decades, the treatment of valvular heart disease has advanced, allowing many individuals with congenital or early acquired heart disease to go on to live full, healthy lives. As a result, women in the reproductive age with a mechanical heart valve (MHV) are increasingly pursuing pregnancy and require tight control of anticoagulation (AC) throughout gestation. ${ }^{1}$

Given the teratogenic risks of vitamin $\mathrm{K}$ antagonists (VKA) to the fetus, ${ }^{2}$ as well as the known thrombogenic and cardiovascular risks to the mother, women with MHVs were, in many cases, previously advised to avoid pregnancy; however, this is no longer the case. If a woman is in an otherwise well-controlled health state with appropriate cardiac function, the physiological changes of pregnancy are usually well tolerated, and AC therapy can be adjusted to minimize embryonic teratogenicity while decreasing maternal thrombogenic risk. Ideally, the pregnancy is planned after discussions with cardiologists and obstetricians, who can review maternal and fetal risks with the woman. Counseling a patient on AC options during the antepartum period requires a discussion of specific risks and benefits of various treatment options, followed by shared decision-making to determine the optimal treatment regimen. As most patients are maintained on VKA outside of pregnancy, fetal risks are critical to review in view of the dose-related response of VKA-mediated embryopathy. ${ }^{1,3,4}$ Despite recommendations for VKA to serve as the firstline antepartum treatment, ${ }^{5}$ low-molecular weight heparin (LMWH) - which does not cross the placenta and is safely used in pregnancy for other AC requirements - is increasingly identified by patients and providers as the antepartum treatment of choice despite concerns on increased maternal risk of valve thrombosis. ${ }^{6,7}$

Even in experienced hands, the management of AC therapy for this high-risk patient population is a challenge. Most of the current obstetric literature focuses on antepartum $\mathrm{AC}$, with guidance for transition to the intrapartum period and limited direction for the early postpartum period. The American College of Obstetrics and Gynecology recommends that AC can be reasonably resumed 4-6 hours after vaginal delivery, and 6-12 hours after a cesarean. ${ }^{8}$ However, these guidelines are directed toward women treated for thromboembolism in pregnancy and are not specific for women with MHV; of note, the optimal time to restart AC therapy in the postpartum period for women with MHV is unclear. This is, in part, due to limited investigation into the initiation of postpartum therapeutic AC in this patient population. ${ }^{9}$

Although $\mathrm{AC}$ is required to prevent valve thrombosis and its sequelae, initiation of therapeutic postpartum AC in this patient population appears to be a particularly high-risk treatment. Vause et al recently reported a population-based descriptive study, which reported that women with MHV in the UK suffer from high rates of maternal mortality and morbidity. ${ }^{10}$ A large number of morbidities stemmed from hemorrhagic complications experienced in the postpartum period; however, there was no detailed description of postpartum AC management in this cohort. ${ }^{10,11}$

There are several important factors that need to be considered with regard to how to best to manage women with MHV during pregnancy; however, there is a paucity of investigation into how best to initiate early postpartum AC in this patient population. We sought to examine our institutional experience on early postpartum initiation of $\mathrm{AC}$ in women with MHV, with the hope of laying the groundwork for evidence-based guidelines for the resumption of postpartum $\mathrm{AC}$ in this high-risk population.

\section{Methods}

A descriptive retrospective analysis was conducted of pregnant women with MHV on AC therapy who delivered at a single institution. This study was approved by the Yale University Human Investigation Committee. Due to the retrospective nature of the study, individual patient consent was not required. The independent review board-approved study period spanned April 1, 2007 to February 1, 2018. The ICD, Ninth and Tenth Revisions, Clinical Modification (ICD-9-CM and ICD-10-CM) diagnosis and procedure codes for mechanical valve, mitral valve replacement, and aortic valve replacement, as well as primary or secondary diagnosis codes for pregnancy and various methods of delivery, were employed to identify patients. The electronic medical record database was implemented between 2011 and 2012; access to electronic records prior to that time was limited and, therefore, paper charts were reviewed.

For inclusion into the study, women had to have an MHV in place in the mitral or aortic position and been on AC therapy during the study period of the recorded pregnancy episode. Patients with bioprosthetic heart valves were excluded. Medical records were reviewed and verified to meet the inclusion criteria.

Outcome variables included AC therapy prior to and during pregnancy, as well as prior to delivery, and was 
identified as VKA, LMWH, or heparin IV infusion. The AC method and timing, postdelivery, of medication reinitiation were recorded. The cohort of pregnancies was then divided into two groups: women who were restarted on AC 6 hours or less after delivery, and women who were reinitiated on AC more than 6 hours postpartum. ${ }^{8}$

Other maternal outcomes recorded included primary and secondary postpartum hemorrhage, wound hematoma, intra-abdominal bleed, vaginal hematoma, need for reoperation or other intervention, valve thrombosis, known cardiac rhythm with management and complications, stroke, length of hospital stay, and maternal death. The timing and indication for delivery were identified.

Fetal and neonatal outcomes were recorded. They included intrauterine fetal growth restriction, intrauterine fetal demise, neonatal birthweight, Apgar scores at 1 and 5 minutes, and neonatal intensive care unit (ICU) admission.

\section{Statistical analysis}

For descriptive analysis, continuous variables were reported as the mean with SD, median, and range. Categorical variables were reported as proportions.

\section{Results}

The initial search identified 42 women with a nonnative heart valve who also had a pregnancy episode recorded during the 10 -year study period at our institution. After a detailed chart review, nine women were confirmed to have an MHV that required $\mathrm{AC}$ during gestation. Other women were primarily excluded due to the temporality of their MHV placement and pregnancy timing - that is, the MVH was implanted after the index pregnancy. The nine women meeting inclusion criteria had 19 pregnancy episodes recorded (Figure 1). Demographics are delineated in Table 1. Of the women with an MHV on AC during gestation, $55.6 \%$ had a mechanical aortic valve and $44.4 \%$ had a mitral valve.

\section{Antepartum and intrapartum AC management}

Of the cohort of 19 pregnancies, five pregnancies did not meet the inclusion criteria and were excluded from the analysis; these included an ectopic pregnancy, a spontaneous abortion at 9 weeks of gestation, and three elective terminations, all of which were completed without operative or hemodynamic complications. The timing and type of $\mathrm{AC}$ resumption after their procedures were not specifically recorded.

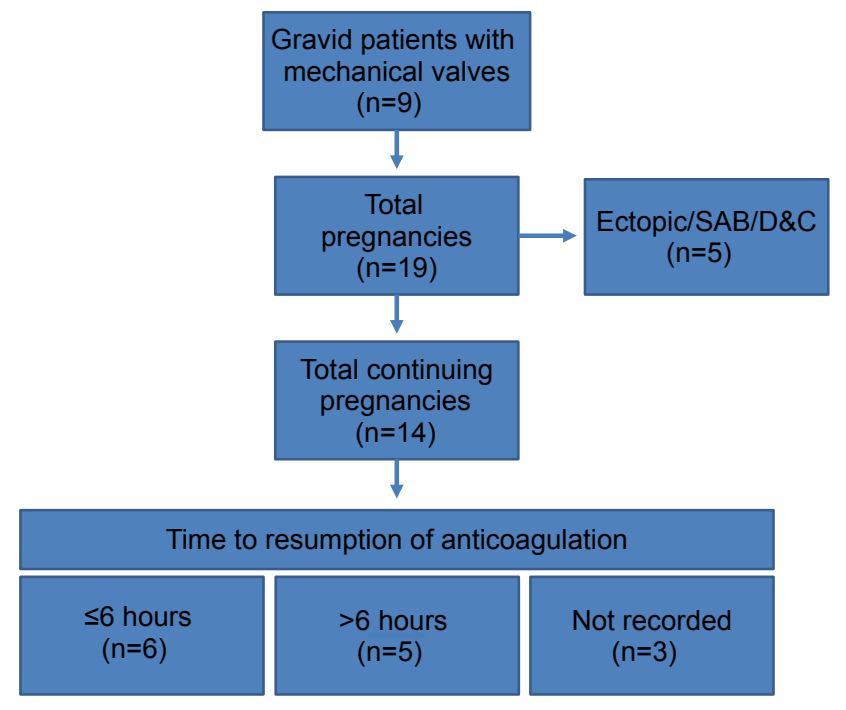

Figure I Flowchart of study population.

Note: Three of the completed gestations did not have the time to anticoagulation resumption recorded after delivery.

Abbreviations: D\&C, dilation and curettage; SAB, spontaneous abortion (miscarriage).

Table I Patient demographics ( $\mathrm{n}=9$ patients)

\begin{tabular}{|c|c|}
\hline Patient characteristic & \\
\hline \multicolumn{2}{|l|}{ Age (years) } \\
\hline Median & 25 \\
\hline Range & $19-39$ \\
\hline \multicolumn{2}{|l|}{ Race (n) } \\
\hline Caucasian & 4 \\
\hline African American & 2 \\
\hline Hispanic & 2 \\
\hline Other & I \\
\hline \multicolumn{2}{|l|}{ Tobacco use (n) } \\
\hline During pregnancy & 3 \\
\hline \multicolumn{2}{|l|}{ BMI $\left(\mathrm{kg} / \mathrm{m}^{2}\right)$} \\
\hline Median & 27.0 \\
\hline Range & $19.3-46.3$ \\
\hline \multicolumn{2}{|l|}{ Valve location (n) } \\
\hline Mitral & 4 \\
\hline Aortic & 5 \\
\hline \multicolumn{2}{|l|}{ Gravidity/parity } \\
\hline Median & $2 / 0$ \\
\hline Range & $4-1 / 1-0$ \\
\hline \multicolumn{2}{|l|}{ Antepartum AC (n) } \\
\hline Warfarin PO & I \\
\hline Heparin SC & 2 \\
\hline Enoxaparin SC & 6 \\
\hline
\end{tabular}

Abbreviations: AC, anticoagulation; BMI, body mass index; PO, by mouth; SC subcutaneous injection. 
Before pregnancy, the majority of women (88.9\%) were therapeutically anticoagulated on warfarin, and one was on LMWH as she was attempting conception and desired to avoid warfarin-induced teratogenicity. During pregnancy, $88.9 \%$ of women who were on warfarin transitioned to LMWH early in the first trimester. One patient remained on VKA throughout gestation due to a known difficulty in achieving therapeutic AC on LMWH.

In late gestation, or during a planned admission immediately prior to delivery, the majority of patients (85.7\%) were transitioned from LMWH to heparin. One patient remained on LMWH until 1 day prior to anticipated delivery. Another patient remained on warfarin during her delivery, as she presented in active labor without time to effectively transition to heparin infusion.

\section{Delivery timing and modes}

Of the delivered pregnancies ( $\mathrm{n}=14$ ), the mean gestational age of delivery was preterm at $35 \mathrm{w} 2 \mathrm{~d}$ (Table 2 ); however, the median was an early term at $37 \mathrm{w} 2 \mathrm{~d}$ (range $28 \mathrm{w} 2 \mathrm{~d}-39 \mathrm{w} 1 \mathrm{~d}$ ). Nine deliveries were via cesarean $(64.3 \%)$, two were vacuum-assisted vaginal (14.3\%), and three were spontaneous vaginal (21.4\%) births. Of the cesarean births, one was emergent due to placental

Table 2 Maternal delivery outcomes ( $n=14$ cases)

\begin{tabular}{|c|c|}
\hline Maternal delivery outcomes & n (\%) or median (range) \\
\hline \multicolumn{2}{|l|}{ GA (w/d) at delivery } \\
\hline Mean & $35 w 2 d$ \\
\hline Median & $37 w 2 d(28 w 2 d-39 w / d)$ \\
\hline \multicolumn{2}{|l|}{ Mode of delivery } \\
\hline Vaginal & $3(2 \mid .4)$ \\
\hline Vacuum-assisted & $2(14.3)$ \\
\hline Cesarean & $9(64.3)$ \\
\hline \multicolumn{2}{|l|}{ Anesthesia } \\
\hline None & I (7.I) \\
\hline Spinal & $3(2 \mid .4)$ \\
\hline Epidural & $5(35.7)$ \\
\hline GETA & $5(35.7)$ \\
\hline \multicolumn{2}{|l|}{ Intrapartum AC } \\
\hline Warfarin PO & I (7.I) \\
\hline Heparin infusion & $12(85.7)$ \\
\hline Enoxaparin SC & I (7.I) \\
\hline \multicolumn{2}{|l|}{ Postpartum AC } \\
\hline Warfarin PO & $\mathrm{I}(7.1)$ \\
\hline Heparin infusion & II (79.6) \\
\hline Enoxaparin SC & $2(14.3)$ \\
\hline
\end{tabular}

Abbreviations: AC, anticoagulation; GA (w/d), gestational age in weeks and days; GETA, general endotracheal anesthesia; PO, by mouth; SC, subcutaneous injection. abruption. An assisted second stage of delivery was planned in one case due to maternal cardiac status.

There were varying modes of analgesia utilized in the deliveries. In total, $21.4 \%$ of deliveries received pain control via regional spinal analgesia, $35.7 \%$ by epidural anesthesia, and $35.7 \%$ through general endotracheal anesthesia. One patient elected to have no medicinal intervention for pain during labor, unrelated to AC status.

\section{Postpartum AC management}

All patients had AC resumption during their hospitalization for delivery. For the entire cohort of delivered pregnancies, the mean time to restart AC after birth was 10 hours (SD 8 hours, range 2-27 hours, median 6 hours; Table 3). Six deliveries $(42.8 \%)$ were followed by resumption of some form of $\mathrm{AC} \leq 6$ hours postpartum (mean 5 hours, SD 2 hours, range 2-6 hours, median 6 hours). In these cases, two patients had mitral MHVs, and four had aortic MHVs. Five deliveries (35.7\%) recorded resumption of AC therapy $>6$ hours postpartum (mean 16 hours, SD 7 hours, range 2-27 hours, median 16 hours). All five cases were in patients with an MHV in the mitral position. Three deliveries did not have the specific time to resumption of AC recorded (Figure 1 and Table 3 ). The anticoagulants employed postpartum were continuous IV heparin (79.6\%), enoxaparin (14.3\%), or warfarin $(7.1 \%)$. There were no instances of combination therapy in the early postpartum period.

\section{Postpartum complications}

Hemorrhagic complications occurred in six of 14 deliveries (42.8\%, Table 3). Three (21.4\%) of the cases were complicated by primary postpartum hemorrhage, and the other three $(21.4 \%)$ were secondary postpartum hemorrhage. The average estimated blood loss at the time of delivery for all patients was $582 \pm 189 \mathrm{~mL}$.

Specifically, an incisional wound hematoma developed in a patient restarted on enoxaparin 27 hours postoperatively. This was managed conservatively and did not require surgical intervention or debridement. An intra-abdominal hematoma occurred on postoperative day 3 in a woman transitioned to intravenous heparin 6 hours postoperatively. Due to hemodynamic instability, surgical intervention was required for clot evacuation and rapid control of active extravasation. Another intra-abdominal bleed occurred in a patient who was reheparinized 4 hours after her cesarean delivery and required embolization of an inferior epigastric artery. Four cases $(28.6 \%)$ required postpartum transfusion of blood products (packed RBCs \pm fresh frozen plasma). 
Table 3 Maternal outcomes. Selected maternal outcomes in the postpartum period were recorded ( $\mathrm{n}=14$ cases). There were no maternal deaths, CVA, or thrombosed valves noted during any of the delivery admissions. One patient suffered a CVA at I6 weeks of gestation but recovered by the time of delivery

\begin{tabular}{|c|c|c|c|}
\hline \multirow[t]{2}{*}{ Maternal outcomes } & \multicolumn{3}{|c|}{ Time to anticoagulation postpartum } \\
\hline & $\begin{array}{l}\text { All deliveries ( } n=14) \\
n(\%) \text {, or median (range) }\end{array}$ & $\begin{array}{l}\leq 6 \text { hours }(n=6) \\
n(\%), \text { or median (range) }\end{array}$ & $\begin{array}{l}>6 \text { hours }(n=5) \\
n(\%), \text { or median (range) }\end{array}$ \\
\hline \multicolumn{4}{|c|}{ Time to $A C$ resumption $P P(n=1 \mid$ recorded) } \\
\hline Median (hours) & $6(2-27)$ & $6(2-6)$ & $16(7-27)$ \\
\hline PP hemorrhage & $6(42.8)$ & $3(50.0)$ & $3(60.0)$ \\
\hline Transfusion & $4(28.6)$ & $2(33.3)$ & $2(40.0)$ \\
\hline Wound hematoma & $2(14.3)$ & 0 & I (20.0) \\
\hline Intra-abdominal bleed & $2(14.3)$ & $2(33.3)$ & 0 \\
\hline Vaginal hematoma & 0 & 0 & 0 \\
\hline Surgical reintervention & I (7.I) & I (I6.6) & 0 \\
\hline Vessel embolization & $I(7.1)$ & $I(16.6)$ & 0 \\
\hline \multicolumn{4}{|c|}{ Length of stay, total hospitalization } \\
\hline Median (days) & $7(3-14)$ & II (6-14) & $4(3-9)$ \\
\hline \multicolumn{4}{|c|}{ Length of stay, postpartum hospitalization } \\
\hline Median (days) & $4(2-10)$ & $5(3-9)$ & $4(2-7)$ \\
\hline Valve thrombosis & 0 & 0 & 0 \\
\hline Stroke & 0 & 0 & 0 \\
\hline Death & 0 & 0 & 0 \\
\hline
\end{tabular}

Abbreviations: AC, anticoagulation; CVA, cerebrovascular accident; PP, postpartum.

The institutional massive transfusion protocol was not employed in any of the cases. In this series, no maternal valve thromboses, strokes, or deaths were recorded.

The mean total length of hospitalization for these patients was 8 days (SD 4 days, median 7 days, range 3-14 days), and the mean length of hospitalization postpartum was 5 days (SD 2 days, median 4 days, range $2-10$ days).

\section{Arrhythmia burden}

At the start of their pregnancies, $57.1 \%$ of the cohort had normal sinus rhythm, none were identified as having atrial fibrillation, and $42.9 \%$ were reported as having some other type of rhythm abnormality (ventricular arrhythmia, ventricular tachycardia, complete heart block, etc.; Table 4). The majority of women $(57.1 \%, \mathrm{n}=8)$ were neither prescribed a medication for their rhythm control during gestation, nor did they have a pacemaker or implantable cardioverter defibrillator in place. In total, $35.7 \%(\mathrm{n}=5)$ of the cohort was medicated antepartum for their arrhythmia and $14.3 \%(\mathrm{n}=2)$ had a device in place (pacemaker or implantable cardioverter defibrillator).

Peripartum rhythm complications did not occur in the majority of cases $(78.6 \%)$. Three women $(21.4 \%)$ were identified as having rhythm complications either antepartum or immediately postpartum. They included ventricular tachycardia requiring admission and additional medications in two cases and atrial flutter requiring cardioversion in another.

\section{Fetal and newborn outcomes}

Of the 19 pregnancy episodes, there were 14 deliveries and 13 livebirths (Table 5). One case of intrapartum intrauterine

Table 4 Maternal rhythm complications ( $n=14$ cases). Other forms of rhythms recorded were ventricular tachycardia and highgrade ventricular arrhythmias. Peripartum rhythm complications included atrial flutter requiring cardioversion and ventricular tachycardia

\begin{tabular}{|c|c|}
\hline Maternal cardiac status & n (\%) \\
\hline \multicolumn{2}{|l|}{ Known rhythm } \\
\hline NSR & $8(57.1)$ \\
\hline Atrial fibrillation & 0 \\
\hline Other & $6(42.9)$ \\
\hline \multicolumn{2}{|c|}{ Antepartum management of cardiac rhythm } \\
\hline Medication & $5(35.7)$ \\
\hline ICD/PM & $2(14.3)$ \\
\hline Observation/expectant & $8(57.1)$ \\
\hline \multicolumn{2}{|c|}{ Peripartum rhythm complications } \\
\hline Yes & $3(21.4)$ \\
\hline No & II (78.6) \\
\hline
\end{tabular}

Abbreviations: ICD, implantable cardioverter defibrillator; NSR, normal sinus rhythm; PM, pacemaker. 
Table 5 Fetal and neonatal outcomes ( $\mathrm{n}=14$ cases). Selected fetal and neonatal outcomes were recorded for the 14 continuing pregnancies

\begin{tabular}{|l|l|}
\hline Fetal or neonatal outcome & n (\%) \\
\hline Live births & $\mathrm{I} 3(92.8)$ \\
\hline Intrauterine fetal demise & $\mathrm{I}(7.2)$ \\
\hline Median birth weight & $3,020 \mathrm{~g}$ \\
\hline Intrauterine growth restriction & $\mathrm{I}(7.2)$ \\
\hline NICU admission & $5(38.5)$ \\
\hline Apgar $\leq 7$ at I minute & $5(38.5)$ \\
\hline Apgar $\leq 7$ at 5 minutes & $4(30.1)$ \\
\hline
\end{tabular}

Abbreviation: NICU, neonatal intensive care unit.

fetal demise was identified. In this case, there was a known fetal genetic abnormality (Smith-Lemli-Optiz syndrome). The majority of the remaining live-born infants $(92.8 \%)$ were appropriately sized for gestational age. There was one case of fetal growth restriction. No structural abnormalities were confirmed in all delivered neonates. Apgar scores of $<7$ were recorded in $38.5 \%$ of newborns at 1 minute, and $30.1 \%$ of newborns at 5 minutes; $38.5 \%$ of newborns required a neonatal ICU admission. There were no identified cases of fetal or neonatal hemorrhage.

\section{Discussion}

The presence of MHV during pregnancy is rare. However, in our series, these women demonstrate a high rate of clinically significant hemorrhagic complications in the immediate postpartum period, warranting evidence-based direction in the management of required therapeutic AC after delivery. The maternal outcomes reported here are consistent with those previously described, where at least $40 \%$ of women suffer some form of major maternal morbidity. ${ }^{10-12}$ However, in these prior reports, the postpartum AC strategies and timing of reinitiation of postpartum AC are only tangentially commented upon, with vague timelines of resumption of AC.

Similar to findings described by Vause et al and Sadler et al, we did not observe any standard approach to intrapartum AC. ${ }^{10-12}$ Women in our study were primarily on LMWH during pregnancy and then transitioned to heparin infusion for both intrapartum and immediate postpartum AC. This management is different from guidelines that recommend the use of VKA in the second and third trimesters of pregnancy. ${ }^{5}$ We recorded no maternal valve thrombosis, stroke, or death in this cohort of women mostly maintained on LMWH throughout gestation. This may be partly attributed to the use of a serial assessment of anti-Xa levels throughout gestation with subsequent LMWH dose adjustment as well as prompt restarting of maternal AC postpartum. ${ }^{6,7,13,14}$ However, the numbers in our cohort are limited.

In our series, the patterns of postpartum $\mathrm{AC}$ management - which we believe was critical to maternal postpartum outcomes - varied widely. The majority (79\%) of postpartum patients were restarted on heparin infusion, which allows for hourly titration dictated by serial partial thromboplastin time levels as well as the ease of reversal in the setting of uncontrolled bleeding. The range of time from delivery to the resumption of therapeutic $\mathrm{AC}$ was quite broad, ranging between 2 and 27 hours. Approximately half of our patients were restarted before or at 6 hours postpartum. When we reviewed the specific adverse maternal postpartum outcomes, two women restarted AC at less 6 hours postpartum experienced significant intra-abdominal bleeding. Half of the $\leq 6$-hour group and $60 \%$ of the $>6$-hour group suffered from obstetrical hemorrhage overall, although no statistical significance could be drawn between the groups, given the limited number of unique patients. Restarting $\mathrm{AC} \leq 6$ hour postdelivery did not increase the incidence of postpartum transfusion in this cohort. Taken together, all women with an MHV have a collective increased risk of major maternal morbidity postpartum, as well as preterm delivery with resultant NICU admission. In our series, there was no observed thrombogenic harm for the group of women who had AC restarted after 6 hours - but before 27 hours - postpartum.

Due to the association between a fibrillation and valve thrombosis, we specifically looked at this parameter in order to assess if our patients were at increased risk of a thrombogenic event. Prepregnancy arrhythmias were noted in $43 \%$ of patients; however, none of them were diagnosed with atrial fibrillation. The majority of women had no peripartum rhythm complications (79\%), whereas $21 \%$ suffered from a peripartum arrhythmia complication necessitating treatment (medical treatment, cardioversion). This observation underscores the need for close cardiac monitoring peripartum with a multidisciplinary team skilled in assessing and treating cardiovascular complications in this population.

Past case series and meta-analyses in the literature have highlighted the maternal thrombogenic risks associated with various $\mathrm{AC}$ methods in pregnancy, which can be mitigated by the prompt resumption of therapeutic $\mathrm{AC}$ in the postpartum period according to published guidelines, ${ }^{8,15}$ and the need for nuanced decision-making with regard to AC choice as it relates to fetal effects. ${ }^{16}$ However, newer literature highlights maternal postpartum risks, especially hemorrhagic complications associated with therapeutic AC in the early 
postpartum period. ${ }^{10}$ The development of severe hemorrhagic complications may limit the use of AC, may increase the need for additional procedures to control bleeding and decrease mobility, and may prolong hospitalization, as reflected in our data where the median total hospitalization and postpartum lengths of stays were extended. All these factors are known to increase the risk of maternal thrombotic sequelae and should be considered.

The 2014 AHA/ACC Valvular Heart Disease Guidelines provide concise, although limited, recommendations for pregnant patients with an MHV in the antepartum period. However, these recommendations do not distinguish between MHV positions - that is, mitral vs aortic - and there are no obstetric-specific guidelines that outline optimal times for restarting postpartum AC. ${ }^{5,17}$ Antepartum, therapeutic AC with frequent monitoring is recommended with the addition of low-dose aspirin in the second and third trimesters. The preferred method of AC is VKA; however, due to the concern of embryopathy in patients requiring higher doses of VKA ( $>5 \mathrm{mg} /$ day), the guidelines recommend the first-trimester use of subcutaneous LMWH at least twice daily or as continuous intravenous unfractionated heparin.

Within the field of cardiovascular surgery, there are no strict guidelines detailing initiation of postoperative AC management for MHV patients, regardless of reproductive status. ${ }^{5}$ Most cardiovascular surgeons and cardiologists managing AC evaluate each case and design an AC strategy based on the patient's individual risks and needs. The position of the valve (the mitral being more thrombogenic than the aortic), arrhythmia burden, left ventricular function, and overall cardiovascular status are considered in AC planning. In consultation with cardiology and cardiovascular surgery, it seems prudent for the obstetric team to similarly individualize the care of each gravid MHV patient.

The number of pregnancies in women with mechanical valve replacement remains low; however, the development of improved cardiac therapies for children and young adults has resulted in a growing population of reproductive women with cardiovascular disease who desire pregnancy. Women with MHV are a high-risk cohort requiring special consideration; a single management algorithm cannot be applied to all. Prior to pregnancy, the ultimate decision to proceed with an MHV should be based on an interdisciplinary conversation between the patient, her cardiologists, and cardiothoracic surgeon, reflecting on the patient's age, potential need or risk for reintervention, and reproductive and lifestyle preferences, while taking into account the risks related to lifelong $\mathrm{AC}$ requirements.
More nuanced recommendations with regard to optimal early postpartum AC for women with MHV are needed. These recommendations should not only take into consideration the mode of delivery and postpartum bleeding risk, but weigh factors such as valve location and type, cardiac function, and arrhythmia burden. Importantly, women with $\mathrm{MHV}$ in pregnancy should be managed by a coordinated maternal cardiac team that includes cardiologists, obstetricians, and anesthesiologists skilled at managing MHV in pregnancy. Knowledge of increased risk for bleeding complications should prompt close postpartum surveillance and management when reinitiating $\mathrm{AC}$.

Although the data collection spanned a 10 -year period, given the rarity of mechanical valve usage in conjunction with parturition, our retrospective cohort is small. Additional limitations of the study include lack of management details for some pregnancies. This finding is attributed to the non-uniform methodology in recording the exact time that $\mathrm{AC}$ is reinitiated. The rarity of the event, coupled with the inconsistencies of documentation, highlights the need for a standard to collect information so that these women have clear plans to disseminate to their medical teams.

Incorporating strategies from other surgical disciplines, pooling obstetric outcome data, and reviewing the practice patterns of referral institutions will be paramount to generate evidence-based guidelines for AC management in the critical immediate postpartum period in the at-risk group of gravid women with MHV. Initiation of AC after delivery should minimize competing risks and sequelae of valve thrombosis and obstetrical hemorrhage.

\section{Disclosure}

The authors report no conflicts of interest in this work.

\section{References}

1. Sillesen M, Hjortdal V, Vejlstrup N, Sørensen K. Pregnancy with prosthetic heart valves -30 years' nationwide experience in Denmark. Eur J Cardiothorac Surg. 2011;40(2):448-454.

2. Iturbe-Alessio I, Fonseca MC, Mutchinik O, Santos MA, Zajarías A, Salazar E. Risks of anticoagulant therapy in pregnant women with artificial heart valves. N Engl J Med. 1986;315(22):1390-1393.

3. Hung L, Rahimtoola SH. Prosthetic heart valves and pregnancy. Circulation. 2003;107(9):1240-1246.

4. Elkayam U, Singh H, Irani A, Akhter MW. Anticoagulation in pregnant women with prosthetic heart valves. J Cardiovasc Pharmacol Ther. 2004, 9(2):107-115.

5. American College of CardiologyAmerican College of Cardiology/ American Heart Association American Heart Association, et al. 2014 $\mathrm{AHA} / \mathrm{ACC}$ guideline for the management of patients with valvular heart disease: a report of the American College of Cardiology/American Heart Association Task Force on Practice Guidelines. J Thorac Cardiovasc Surg. 2014;148(1):e1-e132. 
6. McLintock C, McCowan LM, North RA. Maternal complications and pregnancy outcome in women with mechanical prosthetic heart valves treated with enoxaparin. BJOG. 2009;116(12):1585-1592.

7. Snape E, Thachil J, Clarke B, Vause S. Anti-Xa based dose changes during low molecular weight heparin anticoagulation for mechanical prosthetic heart valves during pregnancy. J Obstet Gynaecol. 2018;38(5): 721-722.

8. James A; Committee on Practice Bulletins-Obstetrics. Practice bulletin no. 123: thromboembolism in pregnancy. Obstet Gynecol. 2011;118: 718-729.

9. Freedman RA, Bauer KA, Neuberg DS, Zwicker JI. Timing of postpartum enoxaparin administration and severe postpartum hemorrhage. Blood Coagul Fibrinolysis. 2008;19(1):55-59.

10. Vause S, Clarke B, Tower CL, Hay C, Knight M. (on behalf of UKOSS). Pregnancy outcomes in women with mechanical prosthetic heart valves: a prospective descriptive population based study using the United Kingdom Obstetric Surveillance System (UKOSS) data collection system. BJOG. 2017;124(9):1411-1419.

11. Vause S, Clarke B, Tower C, Hay C, Knight M. Mechanical prosthetic heart valves (MPHV) in pregnancy are associated with a high risk of maternal and fetal morbidity and mortality. Heart. 2017;103(19):1557.

12. Sadler L, McCowan L, White H, Stewart A, Bracken M, North R. Pregnancy outcomes and cardiac complications in women with mechanical, bioprosthetic and homograft valves. BJOG. 2000;107(2):245-253.
13. Fox NS, Laughon SK, Bender SD, Saltzman DH, Rebarber A. Anti-factor Xa plasma levels in pregnant women receiving low molecular weight heparin thromboprophylaxis. Obstet Gynecol. 2008;112(4):884-889.

14. Sephton V, Farquharson RG, Topping J, et al. A longitudinal study of maternal dose response to low molecular weight heparin in pregnancy. Obstet Gynecol. 2003;101(6):1307-1311.

15. European Society of Gynecology (ESG) Association for European Paediatric Cardiology (AEPC) German Society for Gender Medicine (DGesGM), et al. ESC Guidelines on the management of cardiovascular diseases during pregnancy: the Task Force on the Management of Cardiovascular Diseases during Pregnancy of the European Society of Cardiology (ESC). Eur Heart J. 2011;32(24):3147-3197.

16. Steinberg ZL, Dominguez-Islas CP, Otto CM, Stout KK, Krieger EV. Maternal and fetal outcomes of anticoagulation in pregnant women with mechanical heart valves. J Am Coll Cardiol. 2017;69(22): 2681-2691.

17. Nishimura RA, Otto CM, Bonow RO. 2017 AHA/ACC Focused Update of the 2014 AHA/ACC Guideline for the management of patients with valvular heart disease: a report of the American College of Cardiology/ American Heart Association Task Force on clinical practice guidelines. J Am Coll Cardiol. 2017;70(2):252-289.
International Journal of Women's Health

\section{Publish your work in this journal}

The International Journal of Women's Health is an international, peerreviewed open-access journal publishing original research, reports, editorials, reviews and commentaries on all aspects of women's healthcare including gynecology, obstetrics, and breast cancer. The manuscript management system is completely online and includes

\section{Dovepress}

a very quick and fair peer-review system, which is all easy to use. Visit http://www.dovepress.com/testimonials.php to read real quotes from published authors. 